\title{
ON THE DRAZIN INVERSE AND M-P INVERSE FOR SUM OF MATRICES
}

\author{
YINGYING QIN, ZHIPING XIONG** AND WANNA ZHOU
}

\begin{abstract}
Drazin inverse and M-P inverse have many important applications in the aspects of theoretic research of operator and statistics. In this article, we will exhibit under suitable conditions a neat relationship between the Drazin inverse of $A+B$ and the Drazin inverses of the individual terms $A$ and $B$. Furthermore, with the same thread, we will give an expression of the M-P inverse of $A+B$ in terms of only the M-P inverses of matrices $A$ and $B$.
\end{abstract}

Mathematics subject classification (2010): 15A09, 15A24, 65F05.

Keywords and phrases: Drazin inverse, M-P inverse, group inverse, sum of matrices, rank equality.

\section{REFERENCES}

[1] M. P. Drazin, Pseudoinverses in associative rings and semigroup, Amer. Math. Mothly., 1958; 65: 506-514.

[2] R. Penrose, A generalized inverse for matrix, Proc. Cambridge Philos, Soc., 1955; 51: 406-413.

[3] A. Ben-Israel and T. N. E. Greville, Generalized Inverse: Theory and Applications, WileyInterscience, 1974; 2nd Edition, Springer-Verlag, New York, 2003.

[4] G. WANG, Y. WeI AND S. QIaO, Generalized Inverses: Theory and Computations, Beijing: Science Press; 2004.

[5] R. E. HARTwig AND J. M. SHOAF, Group inverses and Drazin inverses of bidagonal and triangular Toeplitz matrices, Austral. J. Math. Ser. A., 1977; 24: 10-34.

[6] C. D. MEYER AND J. M. SHOAF, Updating finite Markov chains by using technique of group inversion, J. Statist Comput. Simulation., 1980; 11: 163-181.

[7] B. Simeon, C. Fuhrer And P. Rentrop, The Drazin inverse in multibody system dynamics, Numer. Math., 1993; 64: 521-539.

[8] C. R. Rao AND S. K. Mitra, Generalized Inverses of Matrices and its Applications, Wiley, New York; 1971.

[9] T. Ando, Generalized Schur complement, Linear Algebra Appl., 1979; 27: 173-186.

[10] C. R. Johnson, Inverse M-matrices, Linear Algebra Appl., 1982; 47: 195-216.

[11] Z. P. XIONG AND B. Zheng, The reverse order laws for $\{1,2,3\}$ - and $\{1,2,4\}$-inverses of a two matrix product, Appl. Math. Lett., 2008; 21: 649-655.

[12] D. V. Ouellette, Schur complements and statistics, Linear Algebra Appl., 1981; 36: 187-295.

[13] K. JBILOU AND A. MESSAONDI, Matrix recursive interpolation algorithm for block linear systems direct methods, Linear Algebra Appl., 1999; 294: 137-154.

[14] M. Gulliksson, X. Jin, AND Y. WeI, Perturbation bounds for constrained and weighted least squares problems, Linear Algebra Appl., 2002; 349: 221-232.

[15] R. J. DUFFIn, D. HAZONY, AND N. MORRISON, Network synthesis through hybrid matrices, SIAM J. Appl. Math., 1966; 14: 390-413.

[16] K. E. ERICKSON, A new operation for analyzing series parallel networks, IEEE Trans. Circuit Theory CT-6, 1959; 124-126.

[17] R. E. CLINE, Representations of the generalized inverse of sums of matrices, SIAM. J. Numer. Anal., 1965; 2: 99-114.

[18] K. Radoslaw and K. Krezsztof, Generalized inverses of a sum of matrices, Sankhya Ser. A., 1994; 56: 458-464. 
[19] N. Minamide, An extension of the matrix inversion lemma, SIAM J. Algebra. Discrete. Math., 1985; 6: 371-377.

[20] Y. TIAn, The Moore-Penrose inverse for sums of matrices under rank additivity conditions, Linear and Multilinear Algebra, 2005; 53: 45-65.

[21] Z. P. XIONG AND Y. Y. QIN, The weigheed Moore-Penrose inverse for sum of matrices, Operator and Matrices, 2014; 8: 747-757.

[22] Z. P. Xion, Y. Y. QIN AND B. ZHENG, The least square g-inverse for sum of matrices, Linear and Multilinear Algebra, 2013; 61: 448-462.

[23] R. E. HaRTwig, G. R. WANG, AND Y. WeI, Some additive results on Drazin inverse, Linear Algebra Appl., 2001; 322: 207-217.

[24] Y. WEI, On the perturbation bounds for the group inverse and the oblique projection, Appl. Math. Comput., 1999; 98: 29-42.

[25] J. A. FILL AND D. E. FishKIND, The Moore-Penrose generalized inverse for sums of matrices, SIAM J. Matrix. Anal. Appl., 2000; 21: 629-635.

[26] L. MinaLyFFy, An alternative representation of the generalied inverse of partitioned matrices, Linear Algebra Appl., 1971; 4: 95-100.

[27] G. Marsaglia And G. P. Styan, Equalities and inequalities for ranks of matrices, Linear and Multilinear Algebra, 1974; 2: 269-292.

[28] Y. TIAN, Reverse order laws for generalized inverses of multiple matrix products, Linear Algebra Appl., 1994; 211: 85-100. 